\title{
ON $H$-CLOSED AND MINIMAL HAUSDORFF SPACES $\left({ }^{1}\right)$
}

\author{
BY \\ JACK PORTER AND JOHN THOMAS
}

1. Introduction. Properties closely related to compactness have long been of interest to topologists. In this paper we will consider a collection of properties related to the observation that if a compact Hausdorff space $X$ is embedded in a Hausdorff space $Y$, the image of $X$ is always a closed subspace of $Y$. This property was first investigated by Alexandroff and Urysohn [2] in 1924, and such spaces are called $H$-closed. In 1940, Katětov [13] studied minimal Hausdorff spaces and observed that these are precisely the spaces which are $H$-closed and semiregular.

Bourbaki [9] noted that the $H$-closed property (called by him "absolutely closed") is equivalent for a Hausdorff space $X$ to this property:

$H(i)$ Every open filter base on $X$ has a nonvoid adherence;

and that the minimal Hausdorff property is equivalent to this property:

$H(i i)$ Every open filter base which has a unique adherent point converges to that point.

Recently, Scarborough and Stone [19] studied non-Hausdorff spaces which satisfy properties $H(\mathrm{i})$ and $H(\mathrm{ii})$.

A family of sets whose union is dense in $X$ will be called a proximate cover of $X$. Alexandroff observed that a Hausdorff space is absolutely closed if and only if every open cover of $X$ has a finite proximate subcover. If we remove the Hausdorff hypothesis in this characterization of absolutely closed, we get a class of spaces which we will call quasi $H$-closed in analogy to the Bourbaki usage of the term quasi compact. These spaces are studied in $\S 2$ of this paper, and it is noted that they are identical to the $H$ (i) spaces of Scarborough and Stone.

In [13], Katětov showed that any Hausdorff space $X$ could be densely embedded in an $H$-closed space $X^{*}$ having the property that each bounded, real valued continuous function on $X$ can be extended to $X^{*}$. $X^{*}$ is the Katětov extension of $X$. Although $X^{*}$ is the $H$-closed analogue of the Stone-Čech compactification, it is compact only if $X$ is compact. Banaschewski [6] introduced the notion of a projective maximum in a set $\mathfrak{A}$ of extensions of $X$; an extension $Y$ in $\mathfrak{A}$ is a projective maximum in $\mathfrak{A}$ if for each $Z$ in $\mathfrak{A}$ there is a continuous function from $Y$ onto $Z$

Presented to the Society, January 27, 1967; received by the editors October 31, 1966 and, in revised form, April 3, 1968.

(1) A portion of this paper is a part of a doctoral dissertation prepared at New Mexico State University under the supervision of the second author. A portion of the research reported in this paper was done while the first author was the recipient of a National Science Foundation Research Participation Award in Mathematics at the University of Oklahoma during the summer of 1966. 
which leaves $X$ pointwise fixed. In $\S 3$ we prove an analogue of Urysohn's lemma for $H$-closed Urysohn spaces (a space is Urysohn if distinct points have disjoint closed neighborhoods). In $\S 4$ we show that the Katětov extension is a projective maximum in the class of $H$-closed extensions, answering a question raised by Alexandroff in [1]. We are informed by the referee that this result has been obtained independently by C. T. Liu in essentially the same manner. Since Liu's paper will no doubt have appeared by the time this is in print, we have, at the referee's suggestion, omitted most of the details of this proof. In $\$ 5$ we develop the relationship between the operations of forming the Katětov extension and forming the semiregularization of a topological space. Also, in $\S 5$ a question by Berri [7] as to whether every Hausdorff space can be embedded in a minimal Hausdorff space is answered in the affirmative. We are informed by the referee that G. Strecker, in his dissertation, has shown by a different argument that every Hausdorff space can be embedded as a closed subspace of a minimal Hausdorff space.

Banaschewski [3] and [5] has shown that among completely regular spaces only the compact spaces have the Stone-Weierstrass property and that a noncompact, completely regular space can be densely embedded in another noncompact space which has the Stone-Weierstrass property. This extension is a subspace of the Katětov extension. These results motivated Alexandroff [1] to ask whether the Katětov extension has the Stone-Weierstrass property; and motivated Ponomarev $[1$, p. 54] to ask whether every noncompact completely regular space has a noncompact $H$-closed extension with the Stone-Weierstrass property. These questions are answered in $\S 6$. We are indebted to the referee for calling our attention to [10] in which Flachsmeyer obtains (by entirely different methods) essentially the same answers to the questions of Ponomarev and Alexandroff. Our Theorem 6.8 is a bit stronger than Flachsmeyer's Satz 18 which assumes semiregularity.

Concerning notation, we will use $\tau(A)$ to denote the closure of $A$ in the topology $\tau$ on the set $X$, in place of the more common symbol $\bar{A}$, whenever more than one topology on $X$ is under consideration. If $A \subset B \subset C$, we will denote by $\tau_{B}$ the relative topology induced by $\tau$ on $B$, hence $\tau_{B}(A)$ will denote the closure of $A$ in $B$. Similarly, the symbols $A^{\circ}$ and $\operatorname{int}_{\tau}(A)$ will denote the interior of $A$; and $\alpha(A)$ and $\alpha_{\tau}(A)$ will denote the interior of the closure of $A$. For any subset $A$ of $X, \tau\langle A\rangle$ denotes the set of $\tau$-open neighborhoods of $A$. (For a point $x$ of $X, \tau\langle x\rangle \equiv \tau\langle\{x\}\rangle$.) A $\tau$-filter $\mathscr{F}$ is a filter in the lattice $\tau$, that is, a nonvoid subfamily of $\tau$ such that (1) $A, B \in \mathscr{F}$ $\Rightarrow A \cap B \in \mathscr{F}$ and (2) $A \in \mathscr{F}, B \in \tau, B \supset A \Rightarrow B \in \mathscr{F}$. A free filter is a filter with void adherence.

2. Quasi $H$-closure and semiregularity. This section is basically a catalog of useful facts about quasi $H$-closed and semiregular spaces. Proofs are omitted as they are either easily demonstrated or well known.

A space $X$ is quasi $H$-closed if every open cover has a finite proximate subcover. A subspace $A$ of a space $X$ with topology $\tau$ is quasi $H$-closed relative to $X$ if each 
$\tau$-open family which covers $A$ has a finite subfamily whose union is $\tau$-dense in $A$. Unlike compactness, quasi $H$-closure is not an absolute property in that a subspace $A$ of $X$ can be quasi $H$-closed relative to $X$ without being quasi $H$-closed (for example, let $A$ be the points $c_{i}$ and $a$ in [7, Example 1.5]), however, if $A$ is quasi $H$-closed, it is clearly quasi $H$-closed relative to $X$.

(2.1) The closure of a quasi $H$-closed subspace is quasi $H$-closed.

(2.2) In a quasi $H$-closed space, the closure of an open subset is quasi $H$-closed.

(2.3) If $A$ and $B$ are quasi $H$-closed subsets of $X$, so is $A \cup B$.

An unsolved problem is to find a necessary and sufficient condition for a subset of an $H$-closed space to be $H$-closed. It is of course necessary that the subset be closed, and a sufficient condition is given by the next fact.

(2.4) If $A$ is a closed subset of a quasi $H$-closed space $X$, and if the boundary of $A$ is quasi $H$-closed, then so is $A$.

(2.5) Let $X$ be Hausdorff and $A$ be $H$-closed relative to $X$. For each $y$ in $X-A$, there is an open set $U_{y}$ such that $A \subset \tau\left(U_{y}\right) \subset X-\{y\}$. In particular, $A=\bigcap \tau\left(U_{y}\right)$ for $y$ in $X-A$; hence, $A$ is closed in $X$.

A point $p$ of $X$ is a semiregular point if the regular open neighborhoods of $p$ ( $U$ is regular open if $\alpha(U)=U$ ) are a neighborhood base at $p . X$ is semiregular if each point is semiregular. $p$ is a $T_{2}$-point (or $X$ is locally $T_{2}$ at $p$ ) if the intersection of the closed neighborhoods of $p$ is $\{p\}$. Let $\mathfrak{u}(X)$ denote the set of points in $X$ which are the unique adherent points of some $\tau$-filter.

(2.6) $X$ is $H(\mathrm{i})$ if and only if $X$ is quasi $H$-closed.

(2.7) If $X$ is $H(\mathrm{ii})$ and $\mathfrak{u}(X)$ is nonvoid, then $X$ is quasi $H$-closed.

(2.8) If $p \in \mathfrak{u}(X)$, then $\overline{\{p\}}=\{p\}$. If $\mathfrak{u}(X)=X$, then $X$ is $T_{1}$.

(2.9) If $X$ is $H$ (ii), then every $T_{2}$-point of $X$ is a semiregular point.

(2.10) If $X$ is compact, then $X$ is $H$ (ii).

(2.11) If $X$ is quasi $H$-closed and each point of $\mathfrak{u}(X)$ is semiregular, then $X$ is $H($ ii).

The converse of (2.11) is false as the following example shows. Let $X$ be the closed interval $[-1,1]$ of the real line (in the usual topology) with a point $p$ adjoined. The sets $\{\{p\} \cup(0,1 / n) \mid n=1,2, \ldots\}$ are a neighborhood base at $p . X$ is compact and therefore $H($ ii). $\{(-1 / n, 0) \mid n=1,2, \ldots\}$ is an open filter base with 0 as unique adherent point, but $X$ is neither $T_{2}$ nor semiregular at 0 .

(2.12) $H(\mathrm{i})$ is preserved by continuous maps.

Since the family of regular open sets is closed under finite intersections [12] and covers $X$, it is a base for a smaller, semiregular topology $\tau_{s}$ on $X$, called the semiregularization of $\tau$.

(2.13) If $U \in \tau, \tau(U)=\tau_{s}(U)$ and $\alpha_{\tau}(U)=\alpha_{\tau_{s}}(U)$ [9, p. 160].

(2.14) If $U$ and $V$ are disjoint $\tau$-open sets, so are $\alpha(U)$ and $\alpha(V)$ [9, p. 193].

(2.15) If $Y$ is regular and $f: X \rightarrow Y$ is continuous when $X$ has topology $\tau$, then $f$ is continuous when $X$ has topology $\tau_{s}$ [13].

(2.16) If $(X, \tau)$ is $H$-closed (or $H(\mathrm{i}))$, then $\left(X, \tau_{s}\right)$ is minimal Hausdorff (or $H(\mathrm{ii})$ ). 
(2.17) If $(X, \tau)$ is $H$-closed and Urysohn, then $\left(X, \tau_{s}\right)$ is compact [13]; hence, a regular $H$-closed space is compact.

3. H-closed Urysohn spaces. An $H$-closed, Urysohn space need not be compact as illustrated by the subspace $\left\{c_{i}, a_{i j}, a\right\}$ [7, Example 1.5]. Recall that a space is completely Hausdorff if every pair of points can be separated by a continuous function.

THEOREM 3.1. Let $X$ be an $H$-closed Urysohn space, $M$ be $H$-closed relative to $X$ and $p \notin M$. Then one can find neighborhoods of $p$ and of $M$ with disjoint closures.

Proof. The standard argument used to show a compact Hausdorff space is regular can be used to construct disjoint open neighborhoods $U$ of $p$ and $V$ of $M$. Since $\tau(V)$ is quasi $H$-closed relative to $X$ (by (2.2)), the construction may be repeated with $M$ replaced by $\tau(V)$ to complete the proof.

TheOREM 3.2. Let $(X, \tau)$ be an H-closed Urysohn space and A a subset. These are equivalent:

(a) $A$ is $H$-closed relative to $X$,

(b) $X-A$ is $\tau_{s}$-open, and

(c) $A$ is compact in $\left(X, \tau_{s}\right)$.

Proof. The proof is straightforward and is omitted.

TheOREM 3.3. Let $(X, \tau)$ be an H-closed Urysohn space, and $M$ and $N$ be disjoint subsets which are $H$-closed relative to $X$. Then

(a) there is a real-valued continuous function $f$ on $(X, \tau)$ which takes the value 0 on $M$ and 1 on $N$,

(b) $M$ and $N$ have disjoint neighborhoods, and

(c) $(X, \tau)$ is completely Hausdorff.

Proof. (c) and (b) follow from (a) which follows from 3.2(c).

4. Katětov extension. Recall that $(Y, \sigma)$ is an extension of $(X, \tau)$ if $X \subset Y$, $\sigma_{X}=\tau$, and $\sigma(X)=Y$. For any topological property $P$, a $P$-extension is an extension with that property. Let $(Y, \sigma)$ be an extension of $(X, \tau)$. For each $y$ in $Y-X$, let $\mathscr{N}(y)$ be the trace on $X$ of the filter $\sigma\langle y\rangle$, and for $U$ in $\tau$ let

$$
I(U)=\{y \in Y-X \mid U \in \mathscr{N}(y)\} .
$$

This set $I(U)$ will also be denoted by $I(U, \sigma)$ when necessary to single out $(Y, \sigma)$ from other extensions of $X$ which are also being considered.

LeMma 4.1. Let $(Y, \sigma)$ be an extension of $(X, \tau)$ and $U, V \in \tau$.

(a) $I(U) \subset \sigma(U)-X$.

(b) $I(U) \cap I(V)=I(U \cap V)$.

(c) $U \cap V=\varnothing$ if and only if $(U \cup I(U)) \cap(V \cup I(V))=\varnothing$.

(d) For $W \in \sigma, W \subset(W \cap X) \cup I(W \cap X)$. 
(e) $U \cup I(U) \in \sigma$.

(f) For each $W \in \sigma, \sigma(W \cap X)=\sigma(W)$.

Proof. The proof is straightforward and is omitted.

If $Y$ and $Z$ are extensions of $X, Y$ is projectively larger than $Z$ (written $Y>Z$ ) if there is a continuous function from $Y$ onto $Z$ which leaves $X$ pointwise fixed. $Y$ and $Z$ are isomorphic if there is a homeomorphism of $Y$ onto $Z$ which leaves $X$ pointwise fixed. This fact is well known.

(4.2) If $Y$ and $Z$ are Hausdorff extensions of $X$, they are isomorphic if and only if $Y>Z$ and $Z>Y$.

A Hausdorff extension $Y$ of $X$ is a projective maximum in a set $\phi$ of Hausdorff extensions of $X$ if it is projectively larger than each $Z$ in $\phi$. By (4.2), projective maximums, if they exist, are unique.

We now sketch the construction of the Katětov extension [13], as this construction enters into the proofs of subsequent theorems.

Let $X^{*}$ be obtained from the Hausdorff space $X$ by adjoining a set $I(X)$ of "ideal points"- -one point (denoted $p(\mathscr{F})$ ) for each free $\tau$-ultrafilter $\mathscr{F}$. For each $x$ in $X, \tau\langle x\rangle$ is a base for the $\tau^{*}$-neighborhoods of $x$, while a base for the $\tau^{*}$ neighborhoods of the ideal point $p(\mathscr{F})$ is obtained by adjoining $p(\mathscr{F})$ to each set in $\mathscr{F}$. The space $\left(X^{*}, \tau^{*}\right)$ is $H$-closed and contains $X$ as a dense open subset. It is called the Katětov extension of $(X, \tau)$ and is analogous to the Stone-Čech compactification in that every bounded continuous real function on $X$ can be extended to $X^{*}$. For $M$ in $X^{*}$, denote $\operatorname{int}_{\tau^{*}}(M)$ by int* $(M), \alpha_{\tau^{*}}(M)$ by $\alpha^{*}(M), M \cap X$ by $M_{1}$ and $M-X$ by $M_{2}$.

THEOREM 4.3. Let $X^{*}$ be the Katětov extension of $X$.

(a) For $M \subset X^{*}$, int* $(M)=\left(\operatorname{int}_{\tau}\left(M_{1}\right)\right) \cup\left(M_{2} \cap I\left(\operatorname{int}_{\tau}\left(M_{1}\right)\right)\right)$.

(b) For $U$ in $\tau^{*}, \tau^{*}(U)=\tau\left(U_{1}\right) \cup I\left(U_{1}\right)$ and $I\left(U_{1}\right)=\tau^{*}\left(U_{1}\right)-X$.

(c) For $U$ in $\tau, I(U)=I\left(\alpha_{\tau}(U)\right)$.

(d) For $U \in \tau^{*}, \alpha^{*}(U)=\alpha_{\tau}\left(U_{1}\right) \cup I\left(U_{1}\right)$.

Proof. The proof is straightforward and is omitted.

Alexandroff [1, p. 52] wondered if there is a projective maximum among the $H$-closed extensions of a Hausdorff space $X$. Partial results have been obtained in this direction by Fomin [11] and Katětov [14]. The next theorem resolves this problem.

THEOREM 4.4. The Katětov extension $\left(X^{*}, \tau^{*}\right)$ is a projective maximum among the $H$-closed extensions of a Hausdorff space $(X, \tau)$.

Proof. It suffices to show how to construct a continuous function from $X^{*}$ to $h X$, where $(h X, h)$ is any $H$-closed extension of $X$; this amounts to finding, for each $y$ in $h X-X$, the set $\Phi(y)$ of points in $X^{*}-X$ which must map onto $y$. Let $\Phi(y)$ be the set of points $p(\mathscr{F})$ such that $\mathscr{F}$ contains $\mathscr{N}(y)$. One verifies that the sets $\Phi(y)$ 
are never empty and partition $X^{*}-X$, which allows one to construct a function $f$ from $X^{*}$ onto $h(X)$ by sending the points of $\Phi(y)$ onto $y$, and the points of $X$ onto themselves. To check the continuity of $f$, let $W$ be open in $h X$ and let $U=W \cap X$. $f^{-1}(W)$ is equal to $U$ plus the set of ideal points $p(\mathscr{F})$ such that $\mathscr{F} \in \Phi(\dot{y})$ for some $y$ in $W-U$. But then $\mathscr{F}$ contains $U$, so $U \cup\{p(\mathscr{F})\}$ is open and therefore $f^{-1}(W)$ is open.

Corollary 4.5. Let $X$ and $Y$ be Hausdorff spaces, and let $f: X \rightarrow Y$ be a continuous, open function. Then $f$ can be extended to a continuous function $f: X^{*} \rightarrow Y^{*}$.

Note that the Katětov extension is characterized up to isomorphism as the projective maximum $H$-closed extension.

\section{The Katětov extension and its semiregularization.}

THEOREM 5.1. Let $(X, \tau)$ be any topological space. If $\mathscr{F}$ is a (free) $\tau$-ultrafilter on $X$, then $\mathscr{F}_{s}=\mathscr{F} \cap \tau_{s}$ is a (free) $\tau_{\mathrm{s}}$-ultrafilter. If $\mathscr{F}$ and $\mathscr{G}$ are distinct $\tau$-ultrafilters, so are $\mathscr{F}_{s}$ and $\mathscr{G}_{s}$. If $\mathscr{F}^{\prime}$ is a (free) $\tau_{s}$-ultrafilter, then there is a unique (free) $\tau$-ultrafilter $\mathscr{G}$ containing $\mathscr{F}^{\prime}$ and $\mathscr{G}_{s}=\mathscr{F}^{\prime}$.

Proof. The proof is straightforward and is omitted.

Throughout the remainder of this section $(X, \tau)$ will be assumed to be Hausdorff, whence (by (2.14)) $\left(X, \tau_{s}\right)$ is also Hausdorff.

THEOREM 5.2. The identity map $t$ of $X$ can be extended to a continuous, onto, oneto-one function $t^{*}:\left(X^{*}, \tau^{*}\right) \rightarrow\left(X^{\prime}, \tau_{s}^{\prime}\right)$, where $\left(X^{\prime}, \tau_{s}^{\prime}\right)$ is the Katètov extension of $\left(X, \tau_{s}\right)$.

Proof. Define $t^{*}(p(\mathscr{F}))=p\left(\mathscr{F}_{s}\right) . t^{*}$ is one-to-one and onto by Theorem 5.1. To show that $t^{*}$ is continuous, let $U \in \tau_{s}^{\prime}$ and partition $U$ into $U_{1}=U \cap X$ and $U_{2}$ $=U-X$, cf., the notation established in $\S 4$, preparatory to Theorem 4.3. Thus, $U_{1} \in \tau_{s}, U_{2} \subset I\left(U_{1}, \tau_{s}^{\prime}\right)$ and $t^{*-1}(U)=U_{1} \cup t^{*-1}\left(U_{2}\right)$. Note that if the ideal point $p(\mathscr{F})$ belongs to $t^{*-1}\left(U_{2}\right)$, then $p\left(\mathscr{F}_{s}\right)$ belongs to $U_{2}$, whence $\mathscr{F}_{s}$ (and therefore $\mathscr{F}$ ) must contain $U_{1}$. As $U_{1} \cup\{p(\mathscr{F})\}$ is open and $t^{*-1}(U)$ is a union of such sets, it is also open.

Corollary 5.3. Let $U$ be a $\tau_{s}$-open subset of $X$. Then $t^{*}$ maps the set $I\left(U, \tau^{*}\right)$ onto $I\left(U, \tau_{s}^{\prime}\right)$.

Proof. It follows from the proof of Theorem 5.2 that $t^{*}\left(I\left(U, \tau^{*}\right)\right) \supset I\left(U, \tau_{s}^{\prime}\right)$. If $p(\mathscr{F})$ belongs to $I\left(U, \tau^{*}\right)$, then $\mathscr{F}$ contains $U$, and since $U \in \tau_{s}, \mathscr{F}_{s}$ also contains $U$ and therefore $p\left(\mathscr{F}_{s}\right)=t^{*}(p(\mathscr{F}))$ belongs to $I\left(U, \tau_{s}^{\prime}\right)$.

THEOREM 5.4. $t^{*}$ induces a homeomorphism $t_{s}^{*}$ of $\left(X^{*},\left(\tau^{*}\right)_{s}\right)$ onto $\left(X^{\prime},\left(\tau_{s}^{\prime}\right)_{s}\right)$.

Proof. $t_{s}^{*}$ and $t^{*}$ are the same function; hence, $t_{s}^{*}$ is one-to-one and onto. To show that $t_{s}^{*}$ is continuous, let $U=\alpha_{s}^{\prime}(U)$ be a regular open set in $\tau_{s}^{\prime}$. We must show that $t^{*-1}(U)$ is regular open in $\tau^{*}$. By Theorem 4.3(d), an open set $U$ in the Katětov 
extension of $(X, \sigma)$ is regular open if and only if $U_{1}$ is $\sigma$ regular open and $U_{2}$ $=I\left(U_{1}, \sigma^{*}\right)$. Since $t^{*-1}(U)=U_{1} \cup t^{*-1}\left(I\left(U_{1}, \tau_{s}^{\prime}\right)\right)=U_{1} \cup I\left(U_{1}, \tau^{*}\right)$ by Corollary 5.3, and $U_{1}$ is $\tau_{s}$-regular open, hence a priori $\tau$-regular open, we are done. Since $\left(X^{*},\left(\tau^{*}\right)_{s}\right)$ is minimal Hausdorff $(2.16), t_{s}^{*}$ is a homeomorphism.

THEOREM 5.5. Let $i:(X, \tau) \rightarrow\left(X^{*}, \tau^{*}\right)$ and $i_{s}:\left(X, \tau_{s}\right) \rightarrow\left(X^{\prime}, \tau_{s}^{\prime}\right)$ be the usual embedding functions. Let $p:\left(X^{*}, \tau^{*}\right) \rightarrow\left(X^{*},\left(\tau^{*}\right)_{s}\right)$ and $p_{s}:\left(X^{\prime}, \tau_{s}^{\prime}\right) \rightarrow\left(X^{\prime},\left(\tau_{s}^{\prime}\right)_{s}\right)$ be the identity maps. The following diagram is commutative.

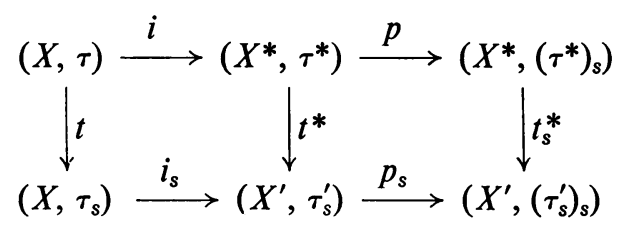

Furthermore, $p_{s} \circ i_{s}$ is a dense embedding, and $t_{s}^{*}$ is a homeomorphism.

Proof. The commutativity of the diagram is easy to check. Since the sets of the form $U_{1} \cup I\left(U_{1}, \tau_{s}^{\prime}\right)$ with $U_{1} \tau$-regular open are a basis of $\left(X^{\prime},\left(\tau_{s}^{\prime}\right)_{s}\right)$ and since the $p_{s} \circ i_{s}$ inverse image of such a set is $U_{1}$, it is clear that $p_{s} \circ i_{s}$ is an embedding. It is a dense embedding since $i_{s}$ is a dense embedding.

COROllary 5.6. $X$ can be densely embedded in a minimal Hausdorff space if and only if $X$ is Hausdorff and semiregular.

Proof. If $X$ is densely embedded in a minimal Hausdorff space, then $X$ is Hausdorff since Hausdorff is a hereditary property. $X$ is semiregular since semiregularity is hereditary on dense subsets [18, Theorem 65]. Conversely, if $X$ is Hausdorff and semiregular, then $p \circ i$ in Corollary 5.6 is a dense embedding since $t$ is the identity homeomorphism.

The last theorem was also obtained by Banaschewski [4] in a different manner. Katětov [16] gives a simple method of embedding any Hausdorff space in a semiregular Hausdorff space, so any Hausdorff space can be embedded in a minimal Hausdorff space. This answers a question posed by Berri in [7].

\section{6. $H$-closed extensions and Stone-Weierstrass property.}

Definition. Let $(X, \tau)$ be Hausdorff and semiregular. Let $\mu X=X^{*}$ and $\mu=\left(t^{*}\right)_{s}$. $(\mu X, \mu)$ will be called the Katětov minimal Hausdorff extension of $(X, \tau)$.

THEOREM 6.1. Let $X$ be completely regular, $\mu X$ the Katětov minimal Hausdorff extension of $X$, and $(\beta X, \beta)$ the Stone-Cech compactification of $X$. There is a continuous, onto function $\phi:(\mu X, \mu) \rightarrow(\beta X, \beta)$ which extends the identity map of $X$.

Proof. By 4.4, there is a continuous, onto function $f:\left(X^{*}, \tau^{*}\right) \rightarrow(\beta X, \beta)$ such that $f(x)=x$ for $x$ in $X$. By $(2.15), \phi:\left(X^{*},(\tau)^{*}{ }_{s}\right) \rightarrow(\beta X, \beta): y \rightarrow f(y)$ is continuous. 
THEOREM 6.2. Let $X$ be completely regular. The following are equivalent:

(a) $\mu X$ is isomorphic to $\beta X$,

(b) $\phi: \mu X \rightarrow \beta X$ is $1-1$,

(c) $\mu X$ is compact, and

(d) $\mu X$ is Urysohn.

Proof. (a) implies (b) is obvious. (b) implies (a) follows from the fact that $\mu X$ is minimal Hausdorff. The equivalence of (c) and (d) follows from (2.17). (a) implies (c) is obvious. (c) implies (a) follows from the well-known fact that any Hausdorff compactification of $X$ which is projectively larger than $\beta X$ is isomorphic to $\beta X$.

If $\mu X$ and $\beta X$ are isomorphic, we will identify them and write $\mu X=\beta X$.

THEOREM 6.3. $\mu X=\beta X$ if and only if $(X, \tau)$ is regular and satisfies the condition that for each pair $\mathscr{F}, \mathscr{G}$ of distinct free $\tau$-ultrafilter, there are open sets $U$ in $\mathscr{F}$ and $V$ in $\mathscr{G}$ such that $\tau(U) \cap \tau(V)=\varnothing$.

Proof. Necessity is obvious. Conversely, suppose $X$ is regular and satisfies the above condition. It suffices to show that $X^{*}$ is Urysohn, for then by (2.17), $\mu X$ is compact. Let $x$ and $y$ be distinct points of $X^{*}$. If one (or both) of the points lie in $X$, it is easy to check that $x$ and $y$ have disjoint closed neighborhoods. Suppose $x=p(\mathscr{F})$ and $y=p(\mathscr{G})$. By hypothesis, there are open sets $U$ in $\mathscr{F}$ and $V$ in $\mathscr{G}$ such that $\tau(U) \cap \tau(V)=\varnothing$, and $U^{\prime}=U \cup\{p(\mathscr{F})\}$ and $V^{\prime}=V \cup\{p(\mathscr{G})\}$ are open neighborhoods of $p(\mathscr{F})$ and $p(\mathscr{G})$. By Theorem 4.3(b), $\tau^{*}\left(U^{\prime}\right)=\tau(U) \cup I(U)$ and $\tau^{*}\left(V^{\prime}\right)=\tau(V) \cup I(V)$. Clearly, $I(U) \cap I(V)$ is void whence $\tau^{*}\left(U^{\prime}\right) \cap \tau^{*}\left(V^{\prime}\right)$ is void.

Definition. Following Katětov [15], we will say that a subset $M$ of $X$ is regularly nowhere dense if there are disjoint open sets $U$ and $V$ such that $\tau(M)=\tau(U) \cap \tau(V)$.

Note that every closed, regularly nowhere dense set is a subset of the boundary of a regular open set, while every boundary of a regular open set is also regularly nowhere dense. Thus the hypothesis that every regularly nowhere dense set is compact can be replaced (as Flachsmeyer [10] does) by the hypothesis that regular open sets have compact boundaries. This condition is satisfied in infinite discrete spaces but not in the real line.

Katětov in [14], [15] proved that $\mu X=\beta X$ if and only if $X$ is completely regular and every regularly nowhere dense closed set in $X$ is compact. He obtained this result through the use of combinatorial embeddings. The next theorem is a direct proof of this fact under the weaker hypothesis that $X$ is regular.

THEOREM 6.4. $\mu X=\beta X$ if and only if $X$ is regular and every regularly nowhere dense closed set in $X$ is compact.

Proof. Suppose $\mu X=\beta X . X$ is clearly regular, and if $M$ is a regularly nowhere dense closed set in $X$, then there are disjoint open sets $U_{1}$ and $U_{2}$ such that $M$ $=\tau\left(U_{1}\right) \cap \tau\left(U_{2}\right)$. For $i=1,2, \alpha_{\tau}\left(X-U_{i}\right)=X-\tau\left(U_{i}\right)$ whence

$$
\left(X-\tau\left(U_{i}\right)\right) \cup I\left(X-\tau\left(U_{i}\right)\right) \in \mu \text {, }
$$


and therefore $W=\bigcup_{i=1}^{2}\left(X-\tau\left(U_{i}\right)\right) \cup I\left(X-\tau\left(U_{i}\right)\right) . X-M$ is a dense open set in $X$ and, hence, belongs to every $\tau$-ultrafilter. Since $X-M=\left(X-\tau\left(U_{1}\right)\right) \cup\left(X-\tau\left(U_{2}\right)\right)$, then $I\left(X-\tau\left(U_{i}\right)\right) \cup I\left(X-\tau\left(U_{2}\right)\right)=\mu X-X$. Thus $W$ is a $\mu$-open set containing $\mu X-X$ and missing $M$. Hence, $\mu(M)=\tau(M)=M$ is compact. Conversely, if $X$ is regular and every regularly nowhere dense closed set in $X$ is compact, it is straightforward to check that the conditions of Theorem 6.3 are satisfied.

COROLLARY 6.5. If $X$ is Hausdorff and semiregular and every regularly nowhere dense closed set in $X$ is compact, then $X$ is completely regular and $\mu X=\beta X$.

Proof. The argument is routine since the hypothesis guarantees that there is a basis of open neighborhoods of each point having compact boundaries.

Katětov [15] proved that, in a Hausdorff space $X$, a necessary and sufficient condition for each nowhere dense closed set in $X$ to be compact is that the subset of nonisolated points of $X$ be compact. Since regularly nowhere dense sets are nowhere dense, then in Hausdorff spaces with a finite number of nonisolated points (in particular, discrete spaces), the Katětov minimal Hausdorff extension is isomorphic to the Stone-Čech compactification.

Definition. Let $C^{*}(X)$ denote the ring of bounded, real-valued continuous functions on $X$. A set $K \subset C^{*}(X)$ is said to separate points if for each pair of distinct points $x$ and $y$ in $X$, there is a function $f$ in $K$ such that $f(x) \neq f(y)$.

Definition. A space $X$ has the Stone-Weierstrass property if $X$ is completely Hausdorff and any subring $K \subset C^{*}(X)$ which separates points has the property that each $f$ in $C^{*}(X)$ is the uniform limit of a sequence of functions in $K$.

LemMa 6.6. $(X, \tau)$ has the Stone-Weierstrass property if and only if $\left(X, \tau_{s}\right)$ has the Stone-Weierstrass property.

Proof. The proof follows from (2.15).

THEOREM 6.7. Let $X$ be H-closed, then the following are equivalent:

(a) $X$ is Urysohn.

(b) $X$ is completely Hausdorff, and

(c) $X$ has the Stone-Weierstrass property.

Proof. Clearly, (c) implies (b) and (b) implies (a). To show (a) implies (c), suppose $X$ is Urysohn, then $\left(X, \tau_{s}\right)$ is compact Hausdorff, and by a theorem of Stone, $\left(X, \tau_{s}\right)$ has the Stone-Weierstrass property. By Lemma 6.6, $X$ has the Stone-Weierstrass property.

In [1] Alexandroff states that it is not known whether the Katětov extension has the Stone-Weierstrass property; the next theorem answers this question not only for completely regular spaces but in complete generality.

THEOREM 6.8. Let $X$ be Hausdorff. The Katětov extension $\left(X^{*}, \tau^{*}\right)$ of $(X, \tau)$ has the Stone-Weierstrass property if and only if every regularly nowhere dense $\tau_{s}$-closed set in $\left(X, \tau_{s}\right)$ is compact. 
Proof. Suppose $\left(X^{*}, \tau^{*}\right)$ has the Stone-Weierstrass property. Let $\left(\mu^{\prime} X, \mu^{\prime}\right)$ be the Katětov minimal Hausdorff extension of $\left(X, \tau_{s}\right)$. By Theorem $5.4,\left(\mu^{\prime} X, \mu^{\prime}\right)$ is homeomorphic to $\left(X^{*},\left(\tau^{*}\right)_{s}\right)$ which is compact by (2.17). By Theorem 6.4, every regularly nowhere dense $\tau_{s}$-closed set in $\left(X, \tau_{s}\right)$ is compact in $\left(X, \tau_{s}\right)$. Conversely, suppose the above condition holds. By Corollary $6.5,\left(\mu^{\prime} X, \mu^{\prime}\right)$ is compact Hausdorff, and by Theorem 5.5, $\left(X^{*},\left(\tau^{*}\right)_{s}\right)$ is compact Hausdorff and, hence, has the Stone-Weierstrass property. By Lemma $6.6,\left(X^{*}, \tau^{*}\right)$ has the Stone-Weierstrass property.

We now proceed to solve the problem proposed by Ponomarev.

Lemma 6.9. Let $X$ be Hausdorff and $X^{*}$ the Katětov extension of $X$. If $U \subset X$ is $\tau$-clopen and $M=\tau^{*}(U)$, then $\left(M,\left(\tau^{*}\right)_{M}\right)$ is the Katětov extension of $U$.

Proof. $\tau^{*}(U)=U \cup I(U)$ in $X^{*}$, and $\tau^{*}(U)$ is $H$-closed by (2.2). Let $\left(U^{*},\left(\tau_{U}\right)^{*}\right)$ be the Katětov extension of $\left(U, \tau_{U}\right)$. For each $p(\mathscr{F}) \in I(U), \mathscr{F}_{U}=\{V \in \mathscr{F} \mid V \subset U\}$ is a free $\tau_{U}$-ultrafilter. Furthermore, each free $\tau_{U}$-ultrafilter $\mathscr{G}$ is contained in a unique free $\tau$-ultrafilter $\mathscr{H}$ and $\mathscr{H}_{U}=\mathscr{G}$. Thus, the function $f: \tau^{*}(U) \rightarrow U^{*}$ defined by $f(x)=x$ for $x$ in $U$ and $f(p(\mathscr{F}))=p\left(\mathscr{F}_{U}\right)$ for $p(\mathscr{F})$ in $I(U)$ is one-to-one and onto. It is straightforward to show that $f$ is continuous. By the comment following Corollary 4.5 , the lemma is established.

Construction 6.10 . Let $(X, \tau)$ be completely regular, and let $\left(X^{*}, \tau^{*}\right)$ be the Katětov extension of $(X, \tau)$. Following Katětov [13], we define the relation $S$ on $X^{*}$ by $p S q$ if and only if for each $f$ in $C^{*}(X), f(p)=f(q)$ where $p, q \in X^{*}$. Let $\rho X$ be the decomposition space $X^{*} / S, \pi: X \rightarrow \rho X$ the natural projection, and $\rho$ the quotient topology $\rho X$ induced by $\pi$. If more than one topology on $X$ is involved, then $(\rho X, \rho)$ will be denoted by $(\rho X, \rho(\tau))$. Using the fact that every $f$ in $C^{*}(X)$ can be extended to $X^{*}$ and the fact that $X$ is completely regular, we see that the only points which get identified in $\rho X$ are points of $X^{*}-X$. For $p(\mathscr{F})$ in $X^{*}-X$, let $S(\mathscr{F})$ denote its equivalence class. The open neighborhood $\{p(\mathscr{F})\} \cup U$ maps onto $U \cup\{S(\mathscr{F})\}$, and this is saturated if and only if $U$ belongs to every free $\tau$-ultrafilter $\mathscr{G}$ for which $p(\mathscr{G})$ belongs to $S(\mathscr{F})$. Thus the sets $\{S(\mathscr{F})\} \cup U$, as $U$ ranges over the largest $\tau$-filter contained in all the $\tau$-ultrafilters equivalent to $\mathscr{F}$, form a neighborhood base of $S(\mathscr{F})$.

THEOREM 6.11. Let $X$ be completely regular and $\rho X$ the space constructed in 6.10 .

(a) $\rho X$ is an $H$-closed, completely Hausdorff extension of $X$.

(b) $\left(\rho X, \rho_{s}\right)$ is isomorphic to $(\beta X, \beta)$.

(c) $X$ is $C^{*}$-embedded in $\rho X$.

(d) $\rho X$ is the projective maximum among $H$-closed, completely Hausdorff extensions of $X$.

(e) If $U \subset X$ is $\tau$-clopen and $M=\rho(U)$, then $\left(M, \rho_{M}\right)$ is isomorphic to $\left(\rho U, \rho\left(\tau_{U}\right)\right)$.

(f) If $X=A \cup B$ where $A, B$ are disjoint, clopen sets, then $(\rho X, \rho)$ is isomorphic to the space which is the topological sum of the spaces $\left(\rho A, \rho\left(\tau_{A}\right)\right)$ and $\left(\rho B, \rho\left(\tau_{B}\right)\right)$. 
Proof. (a), (b), (c) are proven in [13].

Proof of (d). Suppose $(h X, h)$ is an $H$-closed, completely Hausdorff extension of $X$. By Theorem 4.4 there is a continuous, onto function $f: X^{*} \rightarrow h X$. It is straightforward to show that $f \circ \pi^{-1}$ is well defined and continuous.

Proof of (e). Let $N=\tau^{*}(U)$. By Lemma 6.9, it suffices to show that the relation $S$ of 6.10 on $\left(X^{*}, \tau^{*}\right)$ relative to $\left(N, \tau_{N}^{*}\right)$ is the same as the relation of 6.10 (denote this relation by $T)$ placed on $\left(N, \tau_{N}^{*}\right)$. Denote the trace of $S$ on $\left(X^{*}, \tau^{*}\right)$ relative to $\left(N, \tau_{N}^{*}\right)$ by $S_{N}$. If two points of $N$ are separated by a function $f$ in $C^{*}\left(X^{*}\right)$, then $\left.f\right|_{N} \in C^{*}(N)$; so $T \subset S_{N}$. If two points of $N$ are separated by $f$ in $C^{*}(N)$, then define $g$ by $g=\left.f\right|_{U}$ for $x$ in $U$ and $g(x)=0$ for $x$ in $X-U$. Since $U$ is clopen, then $g \in C^{*}(X)$, and $g$ can be extended to $g^{\prime}$ in $C^{*}\left(X^{*}\right)$, and clearly $\left.g^{\prime}\right|_{N}=f$. So $S_{N} \subset T$.

Proof of (f). Every point of $A$ can be separated from every point of $B$ by the real-valued continuous function $g$ which takes the value 0 on $A$ and 1 on $B$ and $g$ can be extended to a $g^{\prime}$ in $C^{*}\left(X^{*}\right)$ [13]. Since every free $\tau$-ultrafilter must contain $A$ or $B, g^{\prime}(p(\mathscr{F}))=0$ or $g^{\prime}(p(\mathscr{F}))=1$. If $A \in \mathscr{F}$ and $p(\mathscr{G}) \in S(\mathscr{F})$, then $A \in \mathscr{G}$; so, $S(\mathscr{F}) \in \rho(A)$ or $S(\mathscr{F}) \in \rho(B)$. This shows that $\rho X$ is isomorphic to the topological sum of $\rho(A)$ and $\rho(B)$. By (e), $\rho X$ is isomorphic to the topological sum of $\left(\rho A, \rho\left(\tau_{A}\right)\right)$ and $\left(\rho_{B}, \rho\left(\tau_{B}\right)\right)$ which establishes (f).

The extension $(\rho X, \rho)$ of 6.10 of a completely regular space is an $H$-closed, completely Hausdorff space and, by Theorem 6.7, has the Stone-Weierstrass property. The next theorem answers the question by Ponomarev.

THEOREM 6.12. The extension $\rho X$ of a completely regular space $X$ is compact if and only if $X$ is compact.

Proof. Suppose $X$ is compact, then $X^{*}=X=\rho X$; hence, $\rho X$ is compact. Conversely, suppose $\rho X$ is compact. Since $X$ is open in $\rho X$, then $X$ is locally compact. If $M$ is a nowhere dense closed subset of $X$, then $X-M$ is an open dense subset of $X$ and, therefore, is contained in every free $\tau$-ultrafilter. Then, $(X-M) \cup\left(X^{*}-X\right)$ is a $\tau^{*}$-open; so, $\rho(M)=\pi\left(\tau^{*}(M)\right)=M$ and $M$ is compact. In [15] Katětov proves that a locally compact space $X$ in which every nowhere dense closed set is compact is the topological sum of a compact space $A$ and a discrete space $B$. Since $\rho X$ is compact, then $\rho X$ is isomorphic to $\beta X$ by Theorem 6.11(b). Since $A$ is compact, then $\rho A=A=\beta A$. By Theorem 6.11(f) and the corresponding theorem for the Stone-Čech compactification, we have that $\rho B$ is isomorphic to $\beta B$. Since $B$ is discrete, then $\rho B=B^{*}$. So, $\beta B$ is isomorphic to $B^{*}$ which happens only if $B$ is finite since the discrete space $B^{*}-B$ is homeomorphic to the compact space $\beta B-B$. Thus $B$ is finite, and since $X=A \cup B, X$ is compact.

\section{REFERENCES}

1. P. Alexandroff, Some results in the theory of topological spaces, obtained within the last twenty-five years, Russian Math. Surveys 15 (1960), 23-83. 
2. P. Alexandroff and P. Urysohn, Zur Theorie der topologischen Räume, Math. Ann. 92 (1924), 258-266.

3. B. Banaschewski, On the Katětov and Stone-Čech extensions, Canad. Math. Bull. 2 (1959), $1-4$.

4. —, Hausdorffsch-minimale Erweiterungen von Räumen, Arch. Math. 12 (1961), 355-365.

5. - On the Weierstrass-Stone approximation theorem, Fund. Math. 44 (1957), 244-252.

6. —_ Extensions of topological spaces, Canad. Math. Bull. 7 (1964), 1-22.

7. M. P. Berri, Minimal topological spaces, Trans. Amer. Math. Soc. 108 (1963), 97-105.

8. N. Bourbaki, Espaces minimaux et espaces complètement séparés, C. R. Acad. Sci. Paris 212 (1941), 215-218.

9. — , Topologie générale, 3rd. ed., Actualités Sci. Indust. No. 1142, Hermann, Paris, 1961.

10. J. Flachsmeyer, Zur theorie der H-abgeschlossen Erweiterungen, Math. Z. 94 (1966), 349-381.

11. S. Fomin, Extensions of topological spaces, Ann. of Math. 44 (1943), 471-480.

12. P. Halmos, Lectures on Boolean algebras, Van Nostrand, Princeton, N. J., 1963.

13. M. Katětov, Über H-abgeschlossene und bikompakte Räume, Casopis Pěst. Mat. 69 (1940), 36-49.

14. — On H-closed extensions of topological spaces, Časopis Pěst. Mat. 72 (1947), 17-32.

15. - On the equivalence of certain types of extension of topological spaces, Casopis Pěst. Mat. 72 (1947), 101-106.

16. —_, A note on semiregular and nearly regular spaces, Časopis Pěst. Mat. 72 (1947), 97-99.

17. A. Ramanathan, Maximal-Hausdorff spaces, Proc. Indian Acad. Sci. Sect. A 26 (1947), 31-42.

18. M. H. Stone, Applications of the theory of Boolean rings to general topology, Trans. Amer. Math. Soc. 41 (1937), 374-481.

19. C. T. Scarborough and A. H. Stone, Products of nearly compact spaces, Trans. Amer. Math. Soc. 124 (1966), 131-147.

UNIVERSITY OF KANSAS,

LAWRENCE, Kansas

New Mexico State University,

University Park, New Mexico 